\title{
OS CRITÉRIOS PARA O USO DE TEXTOS ORIGINAIS NOS TRABALHOS DE CONCLUSÃO DE CURSO DA LICENCIATURA EM MATEMÁTICA DA UECE/ITAPERI (2013 - 2018)
}

\section{THE CRITERIA FOR USING ORIGINAL TEXTS IN THE BACHELOR'S THESES OF UECE'S MATHEMATICS TEACHING UNDERGRADUATE COURSE}

\author{
Laura Damasceno Braga Fontenele ${ }^{1}$; Antonia Naiara de Sousa Batista ${ }^{2}$, \\ Isabelle Coelho da Silva ${ }^{3}$
}

\begin{abstract}
RESUMO
As pesquisas em Educação Matemática estão cada vez mais tratando sobre os recursos e as estratégias que a história da matemática oferece, dentre elas, algumas apresentam importantes debates sobre o uso de textos originais em sala de aula. Diante disso, aflora-se a discussão sobre os critérios para o uso desse material, possibilitando uma articulação entre história e ensino. Assim, este artigo visa descrever um estudo sobre a presença dos critérios para o uso de textos originais no ensino a partir dos Trabalhos de Conclusão de Curso (TCC) da Licenciatura em Matemática da UECE. Para tanto, foi realizada uma pesquisa bibliográfica e documental para investigar a literatura sobre o tema e para coletar os dados dos TCC. Para o período da coleta de dados, delimitamos de 2013 a 2018. Nesse sentido, sete critérios foram levados em consideração na leitura dos dados coletados: a escolha do material; a forma de utilização; a intencionalidade; a série ou o nível escolar; a realização de um tratamento didático; o momento de utilização e a perspectiva historiográfica. Os resultados mostraram que nem todos os critérios foram considerados pelos autores desses TCC, em que os mais constantes foram: a forma de utilizar o material e o objetivo da implementação. Em contrapartida, o critério da escolha da perspectiva historiográfica não foi encontrado em nenhum trabalho. Portanto, inserir textos originais no ensino de Matemática pode ser uma iniciativa em constante crescimento, mas ainda é necessário que os educadores matemáticos conheçam e discutam quais os critérios que eles precisam ter em vista antes de realizar essas propostas na sala de aula.
\end{abstract}

${ }^{1}$ Graduanda em Licenciatura em Matemática pela Universidade Estadual do Ceará (UECE), Bolsista de monitoria da disciplina Estágio Supervisionado I (PROMAC), Fortaleza, Ceará, Brasil. Endereço para correspondência: Av. Dr. Silas Munguba, 1700, Campus do Itaperi, Fortaleza, Ceará, Brasil, país, CEP: 60714-903. E-mail: laura.braga@aluno.uece.br

(iD) ORCID iD: https://orcid.org/0000-0001-5434-0041.

${ }^{2}$ Mestre em Ensino de Ciências e Matemática pelo Instituto Federal de Educação, Ciência e Tecnologia do Ceará (IFCE). Doutoranda do Programa de Pós-Graduação pela Universidade Estadual do Ceará (UECE), Fortaleza, Ceará, Brasil. Endereço para correspondência: Av. Dr. Silas Munguba, 1700, Campus do Itaperi, Fortaleza, Ceará, Brasil, país, CEP: 60714- 903. E-mail: antonia.naiara@aluno.uece.br.

(iD) ORCID iD: https://orcid.org/0000-0003-2305-7088.

${ }^{3}$ Mestre em Ensino de Ciências e Matemática pelo Instituto Federal de Educação, Ciência e Tecnologia do Ceará (IFCE). Doutoranda em Educação Matemática pela Pontifícia Universidade Católica de São Paulo (PUC-SP), São Paulo, São Paulo, Brasil. Endereço para correspondência: Rua Marquês de Paranaguá, 111 - Prédio 1 - $2^{\circ}$ andar, Sala 7, Consolação, São Paulo, São Paulo, Brasil, CEP: 01303-050. Email: isabellecoelhods@gmail.com.

(iD) ORCID iD: https://orcid.org/0000-0002-2936-7143. 
Palavras-chave: Textos originais; Critérios para a utilização de textos originais; Articulação entre história e ensino de matemática.

\begin{abstract}
Research in Mathematics Education is increasingly addressing the resources and strategies that the history of mathematics offers, among them, some present important debates on the use of original texts in the classroom. In view of this, the discussion about the criteria for the use of this material arises, enabling an articulation between history and teaching. Thus, this article aims to describe a study on the presence of criteria for the use of original texts in teaching from the Course Completion Works (TCC) of the Mathematics Degree at UECE. To this end, a bibliographic and documentary research was carried out to investigate the literature on the topic and to collect the data from the CBT. For the period of data collection, we delimited from 2013 to 2018. In this sense, seven criteria were taken into account when reading the data collected: the choice of material; the form of use; intentionality; the grade or school level; carrying out a didactic treatment; the moment of use and the historiographical perspective. The results showed that not all criteria were considered by the authors of these TCC, in which the most constant were: the way to use the material and the purpose of implementation. In contrast, the criterion for choosing the historiographical perspective was not found in any work. Therefore, inserting original texts in the teaching of mathematics can be a constantly growing initiative, but it is still necessary for mathematical educators to know and discuss what criteria they need to keep in mind before making these proposals in the classroom.
\end{abstract}

Keywords: Original texts; Criteria for using original texts; Articulation between history and mathematics teaching. 


\section{Introdução}

A história da matemática é uma área de conhecimento que vem sendo bastante discutida por educadores matemáticos que visam promover a articulação de seus recursos e estratégias com o ensino. Entretanto, muitos pesquisadores ainda consideram a história da matemática como um método ou um recurso pedagógico para o ensino. Traços dessa percepção podem ser vistos na pesquisa de Gutierre (2011, p. 24), em que a história da matemática "pode revelar diferentes métodos para resolver um mesmo tipo de problema, o que, sem dúvida, tem um grande valor pedagógico" e utilizá-la como recurso pedagógico pode proporcionar benefícios tanto na prática do professor quanto na aprendizagem dos alunos.

Porém, outros autores não concordam em reduzir a história da matemática a um recurso ou método. Para D’Ambrósio (1997), Saito (2015) e Roque (2012), ela é uma área de conhecimento que, ao ser articulada com o ensino, proporciona diferentes recursos e estratégias, que podem auxiliar positivamente na aula de matemática e na formação do professor dessa disciplina ${ }^{4}$.

Essa diferença, muitas vezes, está relacionada com a escolha da perspectiva historiográfica adotada em uma pesquisa que envolve a história. Existem diferentes historiografias que delineiam os critérios para a escrita da história e que podem ser classificadas como tradicionais ou atualizadas.

Uma perspectiva historiográfica tradicional considera que a matemática se desenvolveu de forma linear e progressista até o que se tem hoje, que seria o momento mais avançado dessa área. No entanto, uma vertente atualizada acredita que diferentes épocas produziram diferentes formas de saberes, evitando realizar comparações entre elas, pois fazem parte de epistemologias distintas (SILVA, 2018).

A primeira vertente vê a história da matemática como um recurso ou um instrumento para ser utilizado no ensino. Já a segunda considera que ela é uma área de conhecimento e que a sua articulação com o ensino pode promover recursos potencialmente didáticos.

Nesse sentido, uma das formas de se promover essa articulação é através do uso de textos originais. Segundo Silva (2018), esses textos originais são recortes escritos de

\footnotetext{
${ }^{4}$ Vide Alves (2019) e Batista (2018) como exemplos de estudos sobre a articulação entre história e ensino.
} 
documentos históricos, que podem ser estudados, em sala de aula, para se discutir a construção de conceitos e os contextos históricos da época na qual foram desenvolvidos esses conceitos. Nessa conjectura, esses textos podem ser encontrados em documentos originais, que, de acordo com Saito (2015, p. 27), são:

\begin{abstract}
Esses documentos, que são de diferentes campos de conhecimento (portanto, não só matemáticos), são estudados e analisados pelo historiador de acordo com a problemática por ele selecionada. Fazem parte desse conjunto de documentos não só livros e tratados, mas também cartas, manuscritos, minutas e outros documentos não só escritos, mas também aqueles da cultura material, tais como instrumentos, monumentos, máquinas etc.
\end{abstract}

Devido à importância desses materiais, pesquisas vêm sendo realizadas com o sentido de articular a história e o ensino de matemática, como podemos notar em Silva (2018) e Morey (2013). Essas pesquisadoras abordam o uso de documentos históricos e mostram como eles vêm sendo tema de debates nacionais e internacionais cada vez mais constantes.

Contudo, o processo de escolha do texto original até a sua aplicação, em sala de aula, pode não ser um caminho tão simples, visto que existem alguns cuidados a serem tomados antes de apresentá-los aos estudantes em uma proposta para o ensino. Dessa forma, Silva (2018) defende que os educadores matemáticos precisam definir quais os critérios para inserir um original em sala de aula.

Ao escolher um texto original que quer utilizar em sala de aula, assim como em qualquer planejamento que o professor necessita fazer para suas aulas, o educador precisa ter em mente qual é a intencionalidade da implementação desse texto, em qual série ou nível escolar ele pretende fazer essa aplicação, de qual maneira dar-se-á essa realização e se o texto escolhido possui uma linguagem que possa ser compreendida pelos estudantes. Esses são alguns dos critérios elencados por Silva (2018) para o uso desse material em sala de aula e que devem estar bem definidos antes de uma aplicação.

Logo, temos o intuito de descrever a presença dos critérios para o uso de textos originais no ensino a partir dos Trabalhos de Conclusão de Curso (TCC) da Licenciatura em Matemática, da Universidade Estadual do Ceará (UECE), datados entre os anos de 2013 e 2018. É importante ressaltar que este artigo é um recorte de um estudo mais amplo realizado, também, durante o curso de Licencatura em Matemática da mesma instituição. 


\section{Uma breve explanação sobre os critérios de utilização de textos históricos em sala} de aula

Conforme mencionado anteriormente, o estudo dos critérios para o uso de um texto original, em sala de aula, ainda, é pouco debatido entre os educadores matemáticos. Silva (2018) realizou um estudo em que elencou sete critérios, a partir da leitura de textos que envolvem a articulação entre história da matemática e ensino, baseando-se, principalmente, em Massa Esteve et al. (2011), que tinham realizado um estudo preliminar sobre o assunto.

Dessa forma, os sete critérios elencados, foram: a escolha do material; a forma de utilização; a intencionalidade; a série ou o nível escolar; a realização de um tratamento didático; o momento de utilização e a perspectiva historiográfica (SILVA, 2018). A autora ressalta que esses não são os únicos critérios para se utilizar documentos originais, mas foram aqueles encontrados no período de realização de sua pesquisa e com as referências bibliográficas a que ela tinha acesso. Portanto, outros estudos podem encontrar diferentes critérios.

De acordo com Silva (2011), o primeiro critério fala sobre a necessidade de discussão sobre a razão de escolha do documento em que o texto foi recortado e que foi a principal fonte de informação do educador matemático. Enquanto o segundo critério aborda a forma como esse material pode ser utilizado em sala de aula, de modo a promover as suas potencialidades didáticas. Já o terceiro critério frisa a importância da clareza do objetivo de quem se propõe a utilizar um original em sala de aula.

O quarto critério fala sobre a escolha da série ou do nível escolar para o qual a proposta está direcionada, em que o educador deve ter certeza da preparação do seu público para receber e lidar com esse material. O quinto critério questiona se o educador fez algum tratamento didático sobre o texto histórico, a fim de garantir a sua compreensão e adequação ao público-alvo. O sexto critério discute o momento escolhido pelo educador para inserir o original, seja o início, o meio, o fim ou a aula toda. Por fim, o último critério busca saber a perspectiva historiográfica adotada pelo educador matemático para guiar as ações com o original e todas as pesquisas relacionadas à parte histórica (SILVA, 2021).

Nessa perspectiva, a autora realizou um estudo em teses e dissertações que envolvem o uso de originais para o ensino, com a intenção de identificar como os 
pesquisadores brasileiros abordam esses critérios. De forma similar, buscamos, nos TCC defendidos na UECE, entre os anos de 2013 e 2018, quais desses critérios são levados em consideração pelos graduandos que se propõem a pesquisar sobre o uso de textos originais na sala de aula.

\section{Procedimentos metodológicos da pesquisa}

Para a realização deste estudo, inicialmente, foi realizada uma pesquisa bibliográfica, que, segundo Prodanov e Freitas (2013, p. 54), é "elaborada a partir de material já publicado, constituído principalmente de: livros, revistas, publicações em periódicos e artigos científicos, jornais, boletins, monografias, dissertações, teses, material cartográfico, internet”. Diante disso, fizemos um levantamento de pesquisas que abordassem discussões sobre documentos e textos originais, ensino de matemática e história da matemática, a fim de fundamentar teoricamente o estudo realizado.

Além disso, também adentraremos na pesquisa documental, que, conforme Gil (2008), possui como principal diferença, comparada à bibliográfica, a natureza da fonte de pesquisa. Isto é, enquanto a pesquisa bibliográfica fundamenta-se nas contribuições de vários autores que abordam o mesmo assunto, a pesquisa documental baseia-se em materiais que não foram analisados em estudos anteriores ou que, a partir da perspectiva de outros pesquisadores, possam ser abordados de maneiras distintas.

Nesse sentido, os documentos analisados, neste estudo, são os Trabalhos de Conclusão de Curso (TCC) da Licenciatura em Matemática, da UECE, datados entre os anos de 2013 e 2018 e que foram coletados na Biblioteca Central Professor Antônio Martins Filho - Campus Itaperi, em Fortaleza, Ceará. Essa coleta consistiu em duas etapas: na primeira etapa, foram encontrados 108 TCC, cuja busca foi realizada nos computadores disponíveis para pesquisa no acervo da biblioteca, digitando-se a palavrachave "matemática" (entre aspas); na segunda etapa, realizada a partir de uma consulta diretamente com a bibliotecária, foram identificados mais 81 TCC, que não estavam disponíveis na primeira etapa. Assim, nossa investigação foi feita tendo como base 189 Trabalhos de Conclusão de Curso do curso de Licenciatura em Matemática, da Universidade Estadual do Ceará, datados entre os anos de 2013 e 2018. 
Por meio desse material, realizamos uma análise inicial para identificar aqueles TCC que tratavam da História da matemática. Para tanto, fizemos uma leitura panorâmica dos textos, com foco nos seus títulos, palavras-chave, sumários e introduções, encontrando 44 TCC. Dentre eles, apenas 11 pesquisas apresentam documentos históricos, em que alguns visam a aplicação de atividades que possam mobilizar conhecimentos matemáticos na formação de professores de Matemática. Por fim, foi identificado que, desses 11 TCC, 6 tratam de instrumentos históricos e 5 focam no uso de textos originais. Portanto, as investigações realizadas, neste estudo, baseiam-se nesses 5 TCC do curso de Licenciatura em Matemática, da UECE e podem ser conferidas a seguir.

\section{Os critérios utilizados pelos Trabalhos de Conclusão de Curso da Licenciatura em}

\section{Matemática}

A partir de todo o processo realizado anteriormente, selecionamos 5 TCC que focam na utilização de textos originais para o ensino de matemática, de modo a vislumbrar quais critérios elencados por Silva (2021) são manifestados para o uso de textos originais nesses estudos.

Conforme esses critérios, buscamos identificar a preocupação dos autores com a escolha do material que estão propondo em seus estudos; a forma de utilização desses materiais; o objetivo da sua implementação; a série ou o nível escolar em que se pode realizar a aplicação; a existência da necessidade de fazer um tratamento didático; o momento em que se pretende utilizar o texto original escolhido e qual a perspectiva historiográfica adotada pelo autor para estruturar toda a construção da proposta de utilização desse texto original. Para melhor organizar o relato dos dados encontrados, o Quadro 1 mostra esses critérios e os códigos que adotamos para nos referirmos a eles.

Quadro 1 - Critérios para o uso de textos originais e seus respectivos códigos

\begin{tabular}{|c|c|}
\hline CRITÉRIO & CÓDIGO \\
\hline “Qual material utilizar?” & CR1 \\
\hline "Qual a forma de utilizar o material?” & CR2 \\
\hline “Qual o objetivo da implementação?” & CR3 \\
\hline "Em que séries ou nível escolar pode-se aplicar?” & CR4 \\
\hline "Precisa-se fazer um tratamento didático?” & CR5 \\
\hline
\end{tabular}


Laura Damasceno Braga Fontenele, Antonia Naiara de Sousa Batista e Isabelle Coelho da Silva Os critérios para o uso de textos originais nos trabalhos de conclusão de curso da licenciatura em Matemática da UECE/Itaperi (2013 - 2018)

\begin{tabular}{|c|c|}
\hline "Quando utilizar o texto original?" & CR6 \\
\hline "A perspectiva historiográfica escolhida" & CR7 \\
\hline
\end{tabular}

Fonte: Elaborado pelos autores.

No Quadro 2, a seguir, apresentamos os títulos, os autores e o ano de publicação dos cinco TCC em que identificamos a utilização de textos originais para o ensino de Matemática. Além disso, listamos quais são os critérios que percebemos estar presentes nesses TCC, de acordo com os códigos apresentados no Quadro 1.

Quadro 2 - Critérios presentes nos TCC

\begin{tabular}{|c|c|c|}
\hline $\begin{array}{l}\text { TÍTULO DO TRABALHO DE } \\
\text { CONCLUSÃO DE CURSO }\end{array}$ & $\begin{array}{l}\text { AUTOR } \\
\text { (ANO) }\end{array}$ & $\begin{array}{c}\text { CRITÉRIOS PRESENTES NO } \\
\text { ESTUDO }\end{array}$ \\
\hline $\begin{array}{l}\text { O USO DE FONTES HISTÓRICAS PARA O } \\
\text { ENSINO DE MATEMÁTICA: PROPONDO } \\
\text { ATIVIDADES UTILIZANDO O PAPIRO } \\
\text { DE RHIND }\end{array}$ & $\begin{array}{l}\text { Nogueira } \\
(2015)\end{array}$ & CR2; CR3; CR4; CR5; CR6. \\
\hline $\begin{array}{l}\text { O USO DE FONTES NA DISCIPLINA } \\
\text { HISTÓRIA DA MATEMÁTICA A PARTIR } \\
\text { DO PROBLEMA 56 DO PAPIRO DE } \\
\text { RHIND }\end{array}$ & Silva (2016a) & CR1; CR2; CR3; CR5; CR6. \\
\hline $\begin{array}{l}\text { POSSIBILIDADES DO USO DE FONTES } \\
\text { HISTORICAS A PARTIR DOS VERSOS DE } \\
\text { LILAVATI PARA A FORMAÇÃO INICIAL } \\
\text { DOS PROFESSORES DE MATEMÁTICA }\end{array}$ & $\begin{array}{l}\text { Silva } \\
(2016 b)\end{array}$ & CR1; CR2; CR3; CR5; CR6. \\
\hline $\begin{array}{l}\text { A CONCEPÇÃO DOS LICENCIANDOS } \\
\text { EM MATEMÁTICA DA UECE SOBRE O } \\
\text { USO DE FONTES } \\
\text { BISTÓRICAS } \\
\text { REVISTA AL - KARISMI }\end{array}$ & Sousa (2018) & CR1; CR2; CR3. \\
\hline 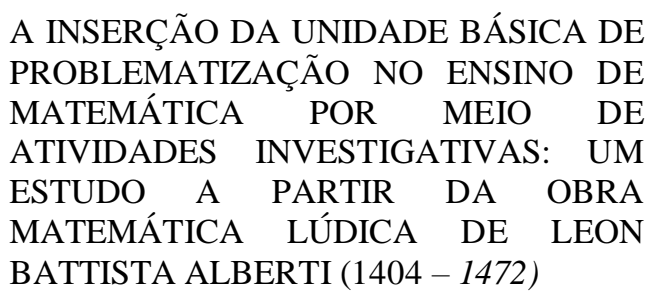 & $\begin{array}{l}\text { Tavares } \\
(2018)\end{array}$ & CR1; CR2; CR3; CR5. \\
\hline
\end{tabular}

Fonte: Elaborado pelos autores.

A seguir, a partir da leitura desses TCC retratados no Quadro 2, apresentamos uma descrição sobre a presença dos critérios para o uso de textos originais no ensino, em estudos realizados por graduandos do curso de Licenciatura em Matemática da UECE. Salientamos que esses trabalhos foram realizados antes do estudo de Silva (2018) e os 
autores, provavelmente, não tiveram acesso a uma literatura que abordasse essa questão, havendo a necessidade de indicar esses critérios.

Assim, o primeiro TCC da nossa coleta foi o de Nogueira (2015). Nesse estudo, a autora propõe desenvolver duas sequências didáticas utilizando o Papiro de Rhind como fonte histórica para o ensino de matemática, em que podemos perceber a presença dos critérios CR2, CR4 e CR6, respectivamente, quando Nogueira (2015) apresenta as sequências didáticas e suas atividades; o seu público-alvo, que é o Ensino Médio e quando se dar-se-á a utilização do texto original escolhido pela autora. O CR3 pode ser identificado quando a autora escreve que o objetivo de sua primeira sequência didática é:

O aluno poderá entender os processos metodológicos utilizados pelos egípcios para a solução dos problemas matemáticos; aprimorar os conhecimentos anteriormente adquiridos sobre volumes por meio da História da matemática; comparar a matemática e os métodos egípcios da antiguidade com a metodologia utilizada a atualmente (NOGUEIRA, 2015, p. 44).

Durante a descrição das suas sequências didáticas, a autora já fornece os problemas traduzidos. Embora ela não cite durante seu estudo, foi realizado um tratamento didático desses problemas, de acordo com a escrita de cada um deles, podendo-se notar a presença do critério CR5.

O próximo estudo selecionado foi o de Silva (2016a). Nessa pesquisa, a autora procura reconhecer a validade do uso do Papiro de Rhind voltado para o ensino de matemática, utilizando o problema 56, que corresponde a um problema sobre o cálculo da inclinação da face da pirâmide. A autora indica que esse papiro foi escolhido para uma aplicação, em sala de aula, por ter sido produzido para o ensino dos escribas, portanto, pode-se notar a presença do CR1.

A investigação proposta por Silva (2016a, p. 40) "foi aplicada em turmas de dois semestres consecutivos, (2015.1 e 2015.2) no curso de Licenciatura em Matemática da UECE”. Entretanto, a autora não deixa clara a razão da escolha desse nível escolar, por isso, não podemos perceber a presença do CR4. Por outro lado, identificamos os critérios CR2 e CR6 quando a autora fala que fez uso de uma atividade e que ela foi precedida de uma aula sobre a matemática egípcia, mostrando que houve um planejamento sobre a utilização do material escolhido e qual seria a melhor forma de fazer esse uso. 
A atividade proposta por Silva (2016a) foi entregue aos alunos contendo o problema na escrita hierática, texto original, que é encontrado no Papiro de Rhind, uma tradução na forma hieroglífica e outra em inglês. Ou seja, um tratamento didático já havia sido realizado sobre o material original e o texto foi entregue aos alunos de forma que eles atingissem os objetivos da proposta, indicando o CR5.

Nessa conjectura, o objetivo era que os estudantes pudessem compreender e explicar a solução do problema como é descrito no papiro, mobilizando as matemáticas existentes nas diferentes culturas (egípcia e moderna). A autora afirma que, previamente, ocorreram aulas sobre a matemática egípcia, tornando melhor a compreensão do método de solução. Com isso, podemos notar a presença dos critérios CR3 e CR5.

O estudo seguinte é o de Silva (2016b), em que o autor propõe a aplicação de uma atividade, que consiste em três versos da obra Lilavati de Bhāskarācārya, fornecido na escrita hindu e, ainda, em uma tradução do texto original para o inglês. Logo, da mesma forma que o TCC anterior, esse mostra uma preocupação com o CR5 e o CR6.

A escolha do material é dada devido ao conteúdo que os versos tratam, tais como o Teorema de Pitágoras e as projeções dos lados de um triângulo qualquer em relação à sua base. Dessa maneira, foi possível perceber os critérios CR1 e CR2. Foi percebida a presença do critério CR3 quando o autor apresenta os objetivos da implementação da atividade proposta, que também são concernentes à compreensão da matemática presente no texto original e à sua relação com a matemática atual.

O TCC de Sousa (2018) foi o próximo estudo selecionado. Nesse trabalho, a autora utiliza, como texto original, as revistas Al-Karismi. A proposta de utilização desse texto acontece devido à importância dessa revista para o ensino de matemática durante o período em que foi publicada. Assim, ela se dá por meio de um curso de extensão universitária, para o qual a autora construiu roteiros didáticos a partir de artigos publicados nela. Logo, podemos perceber a presença dos critérios CR1 e CR2.

Esses artigos contêm uma breve história da origem dos quadrados mágicos e dos conteúdos matemáticos que envolvem números naturais, simetria, sequências numéricas, método de resolução de equações do primeiro e do segundo grau, entre outros. Podemos identificar a presença do CR3 quando Sousa (2018) fala que a escolha dos artigos se deu pelo fato de apresentarem conteúdos de Matemática referentes à educação básica, mais 
especificamente, aos anos finais do Ensino Fundamental. De acordo com Sousa (2018, p. 6), o objetivo era "conhecer as concepções dos licenciandos [...] acerca do uso de fontes históricas, em particular, da Revista Al-Karismi como recurso didático para o ensino, apontando suas potencialidades".

Durante a discussão de seus resultados, a autora indica que os participantes relataram dificuldade em entender a linguagem do texto original utilizado. Além disso, a autora não relata ter sido realizado algum tratamento sobre o material original. Portanto, não identificamos o CR5.

O último estudo selecionado foi o de Tavares (2018), em que a autora propôs um estudo da obra Matemática Lúdica, de Leon Battista Alberti, por meio das Unidades Básicas de Problematização (UBP), como uma ferramenta para a sala de aula, no intuito de proporcionar, ao professor em formação, outros meios que pudessem facilitar a compreensão de conceitos matemáticos da educação básica.

A autora também realizou um curso de extensão, como Sousa (2018), e indicou que a escolha da obra foi por causa de sua existência em Língua Portuguesa e dos conteúdos relacionados à Geometria prática que ela aborda. Portanto, identificamos que a autora se preocupou com os critérios CR1, CR2 e CR3.

Para que os professores em formação pudessem entender a obra histórica Matemática Lúdica, Tavares (2018) afirma que precisou ser realizado um tratamento didático para que a escrita do texto não atrapalhasse a realização da atividade proposta, ou seja, ela levou em consideração o CR5.

Dessa forma, pode-se perceber que, mesmo esses TCC sendo publicados antes do estudo de Silva (2018), nota-se a preocupação dos autores em relação aos procedimentos de uso de textos originais em sala de aula. Isso mostra o quanto esses discentes não estão se formando para a obtenção apenas de um título no ensino superior, mas como professores e pesquisadores, preocupados com a sua prática em sala de aula.

\section{Considerações finais}

O uso de textos originais não é algo novo, mas está cada vez mais presente em discussões que envolvem História da matemática e ensino. Com isso, aumenta, também, a necessidade da presença de critérios que possam guiar as ações dos educadores 
matemáticos que pretendem realizar pesquisas nessa articulação. Esse contexto pode ser percebido no Curso de Licenciatura em Matemática, da UECE, em que os graduandos têm mostrado interesse em realizar pesquisas envolvendo textos originais.

Assim, este artigo buscou descrever a presença dos critérios para o uso de textos originais no ensino nos TCC defendidos por alunos da UECE. Os resultados encontrados mostraram que esses TCC não levam em consideração todos os critérios elencados por Silva (2018), isto é, alguns deles não exploram determinados aspectos importantes em suas propostas para a sala de aula, tais como: o momento de utilização do original; a razão da escolha daquele material; a realização de um tratamento didático etc.

Além disso, o critério sobre perspectiva historiográfica (CR7) não esteve presente em nenhum trabalho, o que mostra que os alunos do Curso de Licenciatura em Matemática, da UECE, que defenderam seus trabalhos entre 2013 e 2018, provavelmente, não conheciam as discussões relacionadas às diferentes perspectivas historiográficas.

Por fim, destacamos que este estudo faz parte de uma pesquisa realizada em um curso de graduação. Portanto, ele não teve a intenção, no momento, de realizar uma análise aprofundada sobre o conteúdo dos TCC e sobre a forma como esses critérios são abordados. Ainda é necessário um estudo mais detalhado desses cinco TCC selecionados, de modo que possamos discutir como esses critérios foram realmente tratados pelos autores, a partir de uma metodologia pertinente para a análise de dados.

\section{Referências}

ALVES, V. B. Um estudo sobre os conhecimentos matemáticos mobilizados no manuseio do instrumento círculos de proporção de William Oughtred. 2019. Dissertação (Mestrado em Ensino de Ciências e Matemática) - Pós-graduação em Ensino de Ciências e Matemática, Instituto Federal de Educação, Ciência e Tecnologia do Ceará, Fortaleza, 2019.

BATISTA, A. N. S. Um estudo sobre os conhecimentos matemáticos incorporados e mobilizados na construção e no uso da balhestilha, inserida no documento Chronographia, Reportorio dos Tempos..., aplicado na formação de professores. 2018. Dissertação (Mestrado em Ensino de Ciências e Matemática) - Pós-graduação em Ensino de Ciências e Matemática, Instituto Federal de Educação, Ciência e Tecnologia do Ceará, Fortaleza, 2018.

D’AMBROSIO, Ubiratan. A era da consciência. São Paulo: Ed. Fundação, Petrópolis, 1997. 
GIL, Antônio Carlos. Métodos e técnicas de pesquisa social. 6. Ed. São Paulo: Altas, 2008.

GUTIERRE, Liliane dos Santos. História da matemática: atividade para a sala de aula. Natal: EDUFRN, 2011. 95 p.

MASSA ESTEVE, Maria Rosa et al. Understanding mathematics using original sources: Criteria and conditions. In: BARBIN, Evelyne; KRONFELLNER, Manfred; TZANAKIS, Constantinos (Ed.). History and Epistemology in Mathematics Education: Proceedings of the 6th European Summer University. Viena: Verlag Holzhausen Gmbh, 2011. p. 415-428

MOREY, Bernadete. Fontes Históricas nas salas de aulas de Matemática: o que dizem os Estudos Internacionais. Revista Brasileira de História da matemática, v. 13, n. 26, p. 73-83. 2013.

NOGUEIRA. Raniele Sampaio. O uso de fontes históricas para o ensino de matemática: propondo atividades utilizando o Papiro de Rhind. Trabalho de Conclusão de Curso - Universidade Estadual do Ceará, Fortaleza, 2015.

ROQUE, Tatiana Marins. História da matemática: Uma Visão Crítica, Desfazendo Mitos e Lendas. $1^{\circ}$ ed. Rio de Janeiro: ZAHAR, 2012.

PRODANOV, Cleber Cristiano; FREITAS, Ernani Cesar de. Metodologia do trabalho científico: Métodos e técnicas da pesquisa e do trabalho acadêmico. 2. ed. Rio Grande do Sul: Universidade Feevale, 2013. 277 p.

SAITO, Fumikazu. História da matemática e suas (re)construções contextuais. São Paulo: Ed. Livraria da Física/SBHMat, 2015.

SILVA. Isabelle Coelho da. O uso de fontes na disciplina história da matemática a partir do problema 56 do Papiro de Rhind. Trabalho de Conclusão de Curso Universidade Estadual do Ceará, Fortaleza, 2016a.

SILVA, Isabelle Coelho da. Um estudo da incorporação de textos originais para a educação matemática: buscando critérios na articulação entre história e ensino. 2018. 92 f. Dissertação (Mestrado) - Curso de Mestrado em Ensino de Matemática, Instituto Federal de Educação, Ciência e Tecnologia do Ceará, Fortaleza, 2018.

SILVA. José Hélison da. Possibilidades do uso de fontes históricas a partir dos versos de Lilavati para a formação inicial dos professores de matemática. Trabalho de Conclusão de Curso - Universidade Estadual do Ceará, Fortaleza, 2016 b.

SILVA, Isabelle Coelho da; Pereira, Ana Carolina Costa. Definições e critérios para uso de textos originais na articulação entre história e ensino de matemática. Boletim de 
Educação Matemática - Bolema [online]. 2021, vol.35, n.69, pp. 223-

241. Epub Apr 16, 2021. ISSN 1980-4415.

https://doi.org/10.1590/1980-4415v35n69a11.

SOUSA. Jeyse Santos de. A concepção dos Licenciandos em Matemática da UECE sobre o uso de fontes históricas brasileiras para o ensino: a revista AL - KARISMI. Trabalho de Conclusão de Curso - Universidade Estadual do Ceará, Fortaleza, 2018.

TAVARES, Marina Oliveira. A inserção da unidade básica de problematização no ensino de matemática por meio de atividades investigativas: um estudo a partir da obra Matemática Lúdica de Leon Battista Alberti (1404 - 1472). Trabalho de Conclusão de Curso - Universidade Estadual do Ceará, Fortaleza, 2018.

Recebido em: 07 / 03 / 2021

Aprovado em: 15 / 04 / 2021 